\title{
Comparative assessment of Jalaukavacharana (Leech Application) and Shringavacharana (Horn Application) in Vicharchika
}

\author{
Research article
}

\author{
Manoj L. Sonaje ${ }^{1}$, Dhiman K. S ${ }^{2}$, Bhuyan $C^{3}$, Gupta S. K ${ }^{4}$, Dudhamal T. S \\ 1. Lecturer, Dept. of Shalya Tantra, Lt.K.R. Ayurveda Medical College, Gadhinglaj, Kolhapur. \\ 2. Professor \& Head Department of Shalakya Tantra, IPGT\&RA, Jamnagar. \\ 3. Prof \& Ex Head, Dept. of Shalya Tantra,IPGT\&RA, Jamnagar. \\ 4. Asso. Professor \& Head Department of Shalya Tantra, IPGT\&RA, Jamnagar. \\ 5. Assistant Professor, Dept. of Shalya Tantra,IPGT\&RA, Jamnagar.
}

\begin{abstract}
Skin disorders are effectively treated in Ayurveda by remedial and Para surgical methods. Vicharchika is a type of Kshudra Kushta; a chronic disease which analogous to Eczema in contemporary medical science. As per modern science, accessible treatment for eczema consists of reassurance, elimination of predisposing causes and palliative measures. Raktamokshana (Blood letting) is one of the precise modality of shodhana (Purification) in skin disorders as it involves vitiated Pitta \& Rakta in its origin. In present study two Raktamokshana methods i.e. Jalaukavacharana (Leech application) and Shringavacharana (Horn Application) were applied with convenient approach and compared for their efficacy in management of Vicharchika. Total 62 patients; 30, 32 patients were treated with Raktamokshana which was done by classical \& adopted innovated modified slant in group A (Jalaukavacharana) and B (Shringavacharana) respectively. Newly innovative Shringavacharana Yantra has been utilized in respective group. Present study shows significant result of Raktamokshana in Vicharchika. Between 2 groups group A (Jalaukavacharana) shows considerable results.
\end{abstract}

Keywords: Vicharchika, Eczema, Jalaukavacharana, Shringavacharana

\section{Introduction}

In Ayurveda every skin disorder is included under heading of Kusta. Ayurveda also implements broad line of management of skin disorders like medicinal and Para surgical approach. Vicharchika is considered as Kshudra Kushta in Ayurvedic classics with elaboration of its management in various aspects.(1) The clinical presentation of

\section{*Correspondence address}

\section{Dr. Manoj L. Sonaje}

202, Saivihar A, Near Krishna Complex, Kathe Lane, Nashik-Pune Road, Dwarka, Nashik. Maharashtra.

E-mail-manojsonaje@gmail.com,

Mob: 09423932283
Vicharchika with symptoms like Kandu (Itching), Srava (Discharge), Pidaka (Pustules), Raji (Scratches), Ruja (Pain), Vaivarnyata (Discoloration of skin) etc.(2) The Eczema is the nearest clinical entity of modern science which can correlate with Vicharchika.(3) Vicharchika shows pathology with vitiation of tridosha but according to law of predominance it shows Pitta \& kapha as major vitiation.(2) The Rakta and Twak is considerably included in pathology as dushya in Vicharchika.(2) All conservative management of Vicharchika has their own limitations with respect to care \& its cure. Acharya Sushruta clearly mentioned that if all conservative management fails then the disease should consider as blood vitiated 
diseases and Raktamokshana is to be considering as choice of treatment.(4)

The existing treatment in modern science for Eczema consists of reassurance, elimination of predisposing causes and palliative measures. It is also said that no specific medication can cure eczema, though few drugs give symptomatic relief only.(5) Modern dermatology employs systemic and local administration of steroids for the management of eczema.(6) Despite an initial response, maintenance therapies with small doses of systemic and topical glucocorticoids usually produce hazardous ill-effects.(7) Similarly electrotherapy, ultraviolet therapy, hydrotherapy, X-ray therapy, etc. are having their own limitations and these therapies are neither popular nor much responding.

Raktamokshana is an effective and safe remedy for the Vicharchika; as major reason for vicharchika is Rakta dushti. Sushruta Samhita details practical guidelines for blood letting which is considered half treatments (Ardha chikitsa)(8)Various methods are employed for blood letting; like Shringa (Horn application), Jalouka (Leech), Alabu(Gourd), Prachhana (Scarification) and Siravyadha (Vein puncture) etc. These therapies are very well advocated in all Ayurvedic texts. Blood letting is effective in all types and stages of Vicharchika. In fact many researches already have been done in this field, but it is need of an hour is to focus on the Raktamokshana therapy to replenish it so that a concrete statement can be made as to which modality helps in which stage of this condition. The surgeon should make use of his yukti according to his experience (Yathabhyasa) to select the disease as well as patient. Raktamokshana should be done according to the procedures (Yathanayaya). (8)

Jalaukavacharana is ancient and famous modality of Raktamokshana which can be used in delicate persons and having pittaghna property. (9) It can be also a choice of treatment where the doshas are deep seated in disease pathologies. (8)

Shringavacharana is the type of Raktamokshana in which cow horn is utilized for the purpose of bloodletting with help of manual mouth aspiration.(9) The acharya Sushruta emphasized the guna of Shringa as Madhura (Sweet), Snigdha (Oily) and Ushna (Hot) which made it vataghana in nature. (9) it is indicated in diseases which are seated at skin level or superficial.(8) Both modalities having their own significance Hence this proposed study was undertaken to evaluate the efficacy and to standardize Jalaukavacharana and Shringavacharana methods of Raktamokshana in Vicharchika.

\section{Aims \& Objective}

To evaluate and compare the efficacy of Jalaukavacharana (Leech Application) and Shringavacharana (Horn Application) in the management of Vicharchika w. s. r. Eczema

\section{Materials and Methods: Study Design}

Present study was randomized, open clinical research at OPD/IPD levels with appropriate sample. The patients included in the clinical trial were divided into two groups viz.

\section{Materials:}

1) Patients - Total 62 patients were divided into two groups, 30 and 32 patients in Group A \& Group B respectively.

2) Raktamokshana Devices:

a. Jalaukavacharana Material - Fresh Jalauka and Turmeric Powder

b. Modified Shringa instrument-* optic fibre tube attached with vacuum pump and pressure regulator. (As Shown in Image 1 to 4) 
- It's a innovative work conducted by scholar first time as a Ph.D. work, The Article has been published in International Journal of Ayurvedic Medicine and Pharmacy: Volume 2, Issue 5, September - October 2011 (IJRAPISSN 2229-3566)

- Group A Treatment of Vicharchika (Eczema) with Jalaukavacharana (Leech Application)

- Group B Treatment of Vicharchika (Eczema) with Shringavacharana (Horn application)

\section{Source of Patients}

Cases of Vicharchika (Eczema) were selected randomly irrespective of their Age, Sex, Religion, Occupation, Caste, Creed etc. and were randomly assigned in all groups, from OPD \& IPD of Department of Shalyatantra I.P.G.T. \& R.A. Hospital, Gujarat Ayurved University, Jamnagar,

\section{Inclusion Criteria}

1) Patients presenting with classical signs and symptoms of Vicharchika (Eczema) like Kandu, Vaivarnyata, Srava, Shotha, Vedana, Pidaka etc.

2) Patients between the age group 1070 years

\section{Exclusion Criteria}

1) Use of Systemic antibiotics or antimycotic drugs in the previous 4 weeks.

2) Known cases AIDS (HIV Positive), Tuberculosis, Anemia and Cardiac Diseases, Leprosy, Hepatitis A, B, C.

\section{Laboratory Investigations}

1) Routine hematological investigations RBS, TLC, DLC, $\mathrm{Hb} \%$, ESR \& PCV, L.F.T., R.F.T, Lipid Profile

2) Urine examination Macroscopic and Microscopic
Methodology

Group A

Modality

Dose:

Period of trial

Diet:

appropriate diet

Procedure:

Patients were given snehana karma before procedure the part was cleaned and fomented with Luke worm water. Then the fresh leeches after activation in the turmeric Powder were applied on the affected part. After application the leech were made to vomit with turmeric powder and the wound was dressed up by pressure bandage. Average $28 \mathrm{ml}$ blood was removed. Duration of Leech application was Average $56 \mathrm{~min}$.

\section{Group B}

Modality

Shringavacharana

Frequency

One sitting per week

Period of trial

Diet

30 days (4 week)

appropriate diet

Procedure Patients were given bahya abhyanga (Local oil Massage) and nadi swedan karma(Sudation) before procedure, small 25 incisions were given at or near the lesion, approximate $1 \mathrm{~mm}$ deapth by 11 no surgical blade were maintained. The innovated Shringa (Optic Tube) was applied from its base over the incised point. The Shringa (Optic Tube) was fixed by pressing it over the skin. The suction was made from the apex of Shringa with vacuum pump with constant $250 \mathrm{~mm}$ of hg pressure. The created vacuum in the Shringa (Optic Tube) removed the vitiated blood up to average $27 \mathrm{ml}$. Average $27 \mathrm{~min}$ was required for procedure. After completion of the procedure the wound was dressed up with antiseptic dressing after haemostasis.

Advice (8)

Do's

1) Laghu ahar (Light Digestive food) 
2) Light Exercise

3) Yavagupan (Use of digestive soups)

\section{Don't's}

1) Anger

2) Hard work

3) Sexual Intercourse

4) Sleeping by day

5) Excessive talking's

6) Physical Exercise

7) Spicy \& Salty food

Assessment Criteria:

Subjective Parameters

1 Kandu (Itching)

2 Vedana (Pain)

3 Vaivarnyata (Discoloration of Skin)

4 Srava (Secretions)

6 Pidaka (Rashes)

7 Rukshata (Dryness)

\section{Objective Parameters}

1 Size of Patches (Calculated the area of Patches in measurement Length $\mathrm{x}$ Breadth $=\mathrm{cm}^{2}$ )

2 Shotha (Swelling) (Calculated by direct measurement in $\mathrm{cm}$ )

\section{Kandu (Itching)}

- No itching 0

- Relive spontaneously +1

- Relive by itching +2

- Disturbs routine +3

- Require medication +4

Vaivarnya (Discolouration)

- Normal colour 0

- Light Brown +1

- Break Brown +2

- Dark Brown +3

- Black Brown +4

Srava (Discharge)

- No discharge 0

- Occasionally discharge +1

- Discharge on itching +2

- Relive spontaneously +3

- Not at all relive +4

Vedana (Pain)

- No pain 0
- Occasionally pain +1

- Mild pain on touch +2

- Mild to moderate pain +3

- Severe pain +4

Pidika (Rashes)

- No Pidaka 0

- Starting of Pidaka +1

- Moderately developed Pidaka +2

- Spreaded over extremities +3

- Severely spreaded all over body +4

Rukshata (Dryness)

- No scratch imprint after scratching 0

- Mild scratch imprint after scratching +1

- Scaling on every scratch +2

- Scratching causes eruption +3

- Spontaneous eruption \& Stretching of skin +4

Follow up

- The follow up of every patient was maintained up to 30 days (Weekly) after completion of treatment.

\section{Statistical Analysis:}

The obtained data were analyzed statistically. Scored values of assessment were analyzed through wilkoxon sign rank test within group and unpaired t test was used for intergroup comparison. For intra group comparison of investigational values paired ' $t$ ' test while intergroup comparison unpaired t test was used. The values were expressed as mean \pm SEM. ' $t$ ' test level of $\mathrm{P}<0.05$ and $\mathrm{P}<0.01$ were considered as statistical significant and highly significant respectively.

Observations and Results:

In present Clinical trial total 63 patients were registered. 31 and 32 patients were in group A, and Group B respectively. Among 63 patients, $71.428 \%$ were male while remaining i.e. $28.572 \%$ patients were female. The maximum number i.e. $44.44 \%$ of patients were in age group of $41-60$ years. The data of Deha Prakriti illustrated that maximum figures of patients i.e. $61.90 \%$ had Vatakapha 
Prakriti, while $14.28 \%$ were of Vata-Pitta Prakriti and $23.82 \%$ patients had PittaKapha Prakriti. As far occupation concerned maximum number of patients was in general official services i.e. $30.158 \%, 12.698 \%$ were in business, $25.396 \%$ were housewives. $92.063 \%$ patients from urban resident \& $7.937 \%$ were from rural areas. Religion showed 92.063\% Hindu and $4.76 \%$ Muslims were observed. On the basis of socioeconomic approach $84.12 \%$ were middle class, $14.28 \%$ were poor and $1.58 \%$ were rich. As per educational status all patients i.e. $100 \%$ were literate. (Table no 1) Vicharchika exhibited $92.063 \%$ as Shushka variety and $7.937 \%$ suffered from Sravi. Presence of the cardinal symptom of Vicharchika like Vaivarnyata (100\%), Kandu (100\%), Pidaka 95.238\%, Raji $100 \%$, Rukshata (100\%) and Ruja $1.587 \%$ proved their prevalence (Table no 2).

The magnificence of present clinical trial showed highly significant consequences in both groups. In Group A cardinal symptoms Kandu, Vaivarnyata, Raji, Rukshata, Size of patches, Pidaka relived significantly showed $\mathrm{p}$ value $<0.001$, other like Vedana, Shotha, Srava were relived but statistically insignificant. (Table no 3) In group B the pacification of cardinal symptoms were also notify highly significant rate as group A (Table no 4). The utmost hematological and biochemical lab investigations showed insignificant changes

\section{Discussion:}

\section{General Observations}

Patient with age group of $41-60$ were up to $44.44 \%$ the reason may by chance. In study maximum $71.428 \%$ patients were of male gender. The male patients are frequently faced the causative factors of skin disorders like cement, mud, coal tar etc due their profession, Tobacco chewing, smoking, alcohol, longstanding are prevalent causes for male involvement in this disease. Maximum numbers of patients had Vata-Kapha Prakriti 61.90\%, but the numbers of the patients in other groups are very nearer to it. Married patients were $76.190 \%$ but the rationality is still unpredictable. Occupational point of view general service men were showed their predominance up to $30.158 \%$ just by chance or the prolonged sedentary posture may causative factor. Socioeconomic approach showed maximum number was in middle class group up to $84.12 \%$, 92.063 exhibited by dwelling status of patients with in urban class just by chance.

\section{Disease Vicharchika}

Amid all Kshudra Kustha Vicharchika is common. The occurrence may be due to climatic condition, because these types of diseases occur chiefly in humid area and the place where this study was carried out is humid region. Vicharchika is a condition where patient presents with the severe painful itching skin eruptions and dryness of the skin which can be rightly correlated to Eczema.

\section{Result}

In leech application the hirudin (Content of Leech saliva) showed rapid action in reliving symptoms $60 \%$ patients got reliving from $2^{\text {nd }}$ sitting.

In Raktamokshana procedures, Shringavacharana was modified; instead of mouth suction vacuum pump was utilized with constant pressure 250-300 $\mathrm{mm}$ of hg. This pressure was estimated threw a serve work of 60 healthy volunteers which showed the average human aspirating capacity is $220 \mathrm{~mm}$ of hg. The dimensionless suction in Shringavacharana procedure got its standardization along with suitable and scientific background. Utilization of vacuum pump avoids aspiration of blood in performer's mouth.

As result concerned in (Group A) Jalaukavacharana \& (Group B) Shringavacharana shows incredible outcome in pacification of symptoms because it is a prime modality of Raktamokshana which can act in any kind 
of vitiation of blood or also beneficial if the vitiation of doshas present systemically.

In group $B$ the pacification of the symptoms are mostly similar to Group A with $p$ value but percentage change shows better results in group A as showed (table no 5).

\section{Conclusion}

The outcome of present clinical trial show highly significant consequences in both groups. In Group A cardinal symptoms Kandu, Vaivarnyata, Raji, Rukshata, Size of patches, Pidaka relived significantly showed $\mathrm{p}$ value $<0.001$, other were relived but remained statistical insignificant. In group B the pacification of cardinal symptoms were also notify highly significant rate as group $\mathrm{A}$ with $\mathrm{p}$ value $<0.001$. The most of hematological and biochemical lab investigations didn't shows remarkable changes The Raktamokshana methods Jalaukavacharana and Shringavacharana are both effective in the management of Vicharchika.

\section{References:}

1. Sushruta, Sushruta Samhita, with Ayurvedtatvasandipika. Kaviraj Dr Ambikadatta Shastri edition 2010. Nidansthan,Varanasi: Chaukhamba Sanskrit Sansthan; 2010.365p.

2. Sushruta, Sushruta Samhita with Nibandhasangraha of Dalhanacharya and Nyayachandrika Panchika of Gayadasa. Yadava T. Nidhana Stana. 7 thed. Varanasi: Chaukhambha
Surabharati Prakashana; 2002. 285, $283 p$.

3. Ronald marks, Roxburgh's common skin diseases, 17th Edition, chapter 8 Eczema (dermatitis) Arnold 2003.107p.

4. Sushruta, Sushruta Samhita, with Ayurvedtatvasandipika. Kaviraj Dr Ambikadatta Shastri edition 2010. Sharirsthana,Varanasi: Chaukhamba Sanskrit Sansthan; 2010.91p.

5. Eczema (Dermatitis), sign and symptoms, available on http://en.wikipedia.org/wiki/Eczema

6. Dr. KK Lo, Medical Bulletin, the Hong Kong Medical Diary, Practical Approach for"Eczema" Vol.15 NO.11 November 2010, Available on http://www.fmshk.org/database/articles /04mb1_5.pdf

7. Dr Haisook's Medicopedia, Cortisol/Glucocorticoids/Corticosteroi ds (actions and side effects), Available on

http://medicopedia.wordpress.com/200 9/01/17/cortisolglucocorticoidscorticos teroids-actions-and-side-effects/

8. Sushruta, Sushruta Samhita with Nibandhasangraha of Dalhanacharya and Nyayachandrika Panchika of Gayadasa. Yadava T. Shareera Sthana. $7^{\text {th }}$ ed. Varanasi:Chaukhambha Surabharati Prakashana; 2004..383, $379 \mathrm{p}$.

9. Sushruta, Sushruta Samhita with Nibandhasangraha of Dalhanacharya and Nyayachandrika Panchika of Gayadasa. Yadava T. Sutra Sthana. $7^{\text {th }}$ ed. Varanasi: Chaukhambha Surabharati Prakashana; 2004. 55,56p. 
Table 1. General observations:

\begin{tabular}{|l|l|l|}
\hline Sr. No. & Observations & Results in \% (Maximum) \\
\hline 1 & Age(41-60years) & 44.44 \\
\hline 2 & Sex (Male) & 71.428 \\
\hline 3 & Marital status (Married) & 76.190 \\
\hline 4 & Occupation (Service) & 30.158 \\
\hline 5 & Dwelling Status (Urban) & 92.063 \\
\hline 6 & Religion (Hindu) & 92.063 \\
\hline 7 & Socio economic status (Middle class) & 84.12 \\
\hline 8 & Educational Status Literate & 100 \\
\hline 9 & Prakriti (Vata-kapha) & 61.90 \\
\hline 10 & Type of Vicharchika & 92.063 \\
\hline
\end{tabular}

Table no. 2 Subjective and Objective Parameters

\begin{tabular}{|l|l|l|l|l|}
\hline Subjective \&Objective Parameters & Present & Present \% & Absent & Absent\% \\
\hline Viavarnyata & 63 & 100 & 00 & 100 \\
\hline Kandu & 63 & 100 & 00 & 100 \\
\hline Srava & 07 & 11.11 & 56 & 88.888 \\
\hline Ruja & 01 & 1.587 & 62 & 98.4127 \\
\hline Pidaka & 60 & 95.238 & 03 & 4.761 \\
\hline Shotha & 00 & 00 & 63 & 100 \\
\hline Rukshata & 63 & 100 & 00 & 00 \\
\hline Raji & 63 & 100 & 00 & 00 \\
\hline
\end{tabular}

Table no 3 Group A Jalaukavacharana Wilcoxon Signed Rank Test

\begin{tabular}{|c|c|c|c|c|c|c|c|}
\hline $\begin{array}{l}\text { Subjective } \\
\text { \&Objective } \\
\text { Parameters }\end{array}$ & $\mathbf{N}$ & Day & Median & $25 \%$ & $75 \%$ & $\mathbf{W}$ & $\mathbf{P}$ \\
\hline \multirow[b]{2}{*}{ Kandu } & 31 & BT $1^{\text {st }}$ Day & 3.000 & 3.000 & 4.000 & \multirow[b]{2}{*}{-496.000} & \multirow[b]{2}{*}{$<0.001 *$} \\
\hline & 31 & $\begin{array}{l}\text { AT } \quad 30^{\text {th }} \\
\text { Day }\end{array}$ & 1.000 & 0.250 & 1.750 & & \\
\hline \multirow[b]{2}{*}{ Vedana } & 31 & BT $1^{\text {st }}$ Day & 0.000 & 0.000 & 0.000 & \multirow[b]{2}{*}{-1.000} & \multirow[b]{2}{*}{1.000} \\
\hline & 31 & $\begin{array}{l}\text { AT } \quad 30^{\text {th }} \\
\text { Day }\end{array}$ & 0.000 & 0.000 & 0.000 & & \\
\hline \multirow[b]{2}{*}{ Size of Patches } & 31 & BT $1^{\text {st }}$ Day & 78.000 & 56.000 & 127.000 & \multirow[b]{2}{*}{-496.000} & \multirow[b]{2}{*}{$<0.001 *$} \\
\hline & 31 & $\begin{array}{l}\text { AT } \quad 30^{\text {th }} \\
\text { Day }\end{array}$ & 60.000 & 40.000 & 94.500 & & \\
\hline \multirow[b]{2}{*}{ Vaivarnyta } & 31 & BT $1^{\text {st }}$ Day & 3.000 & 3.000 & 4.000 & \multirow[b]{2}{*}{-406.000} & \multirow[b]{2}{*}{$<0.001 *$} \\
\hline & 31 & $\begin{array}{l}\text { AT } \quad 30^{\text {th }} \\
\text { Day }\end{array}$ & 1.000 & 1.000 & 2.000 & & \\
\hline \multirow[b]{2}{*}{ Pidaka } & 31 & BT $1^{\text {st }}$ Day & 3.000 & 3.000 & 4.000 & \multirow[b]{2}{*}{-378.000} & \multirow[b]{2}{*}{$<0.001 *$} \\
\hline & 31 & $\begin{array}{l}\text { AT } 30^{\text {th }} \\
\text { Day }\end{array}$ & 1.000 & 1.000 & 3.000 & & \\
\hline \multirow[b]{2}{*}{ Srava } & 31 & BT $1^{\text {st }}$ Day & 0.000 & 0.000 & 0.000 & \multirow[b]{2}{*}{-21.000} & \multirow[b]{2}{*}{0.031} \\
\hline & 31 & $\begin{array}{l}\text { AT } \quad 30^{\text {th }} \\
\text { Day }\end{array}$ & 0.000 & 0.000 & 0.000 & & \\
\hline \multirow{2}{*}{ Shotha } & 31 & BT $1^{\text {st }}$ Day & 0.000 & 0.000 & 0.000 & \multirow{2}{*}{0.000} & \multirow{2}{*}{1.000} \\
\hline & 31 & AT $30^{\text {th }}$ & 0.000 & 0.000 & 0.000 & & \\
\hline
\end{tabular}




\begin{tabular}{|c|c|c|c|c|c|c|c|}
\hline & & Day & & & & & \\
\hline \multirow[b]{2}{*}{ Rukshata } & 31 & BT $1^{\text {st }}$ Day & 3.000 & 3.000 & 4.000 & \multirow[b]{2}{*}{-496.000} & \multirow[b]{2}{*}{$<0.001 *$} \\
\hline & 31 & $\begin{array}{l}\text { AT } \quad 30^{\text {th }} \\
\text { Day }\end{array}$ & 1.000 & 0.000 & 1.750 & & \\
\hline \multirow[b]{2}{*}{ Raji } & 31 & BT $1^{\text {st }}$ Day & 3.000 & 3.000 & 5.000 & \multirow[b]{2}{*}{-351.000} & \multirow[b]{2}{*}{$<0.001 *$} \\
\hline & 31 & $\begin{array}{l}\text { AT } \quad 30^{\text {th }} \\
\text { Day }\end{array}$ & 1.000 & 1.000 & 3.000 & & \\
\hline
\end{tabular}

Table no 4 Group B Shringavacharana Wilcoxon Signed Rank Test

\begin{tabular}{|c|c|c|c|c|c|c|c|c|}
\hline $\begin{array}{l}\text { Subjective } \\
\text { \&Objective } \\
\text { Parameters }\end{array}$ & $\mathbf{N}$ & Day & Median & $25 \%$ & $75 \%$ & $\mathbf{W}$ & $\mathbf{T}+$ & $\mathbf{P}$ \\
\hline \multirow[t]{2}{*}{ Kandu } & 32 & BT $1^{\text {st }}$ Day & 3.000 & 3.000 & 3.000 & \multirow{2}{*}{$\begin{array}{l}- \\
528.000\end{array}$} & \multirow[t]{2}{*}{0.000} & \multirow[t]{2}{*}{$<0.001 *$} \\
\hline & 32 & $\begin{array}{l}\text { AT } \quad 30^{\text {th }} \\
\text { Day }\end{array}$ & 1.000 & 0.000 & 1.000 & & & \\
\hline \multirow[t]{2}{*}{ Vedana } & 32 & BT $1^{\text {st }}$ Day & 0.000 & 0.000 & 0.000 & \multirow[t]{2}{*}{-3.000} & \multirow[t]{2}{*}{0.000} & \multirow[t]{2}{*}{0.500} \\
\hline & 32 & $\begin{array}{l}\text { AT } \quad 30^{\text {th }} \\
\text { Day }\end{array}$ & 0.000 & 0.000 & 0.000 & & & \\
\hline \multirow[t]{2}{*}{ Size of Patches } & 32 & BT $1^{\text {st }}$ Day & 71.000 & 54.000 & 240.000 & \multirow{2}{*}{$-\overline{528.000}$} & \multirow[t]{2}{*}{0.000} & \multirow[t]{2}{*}{$<0.001 *$} \\
\hline & 32 & $\begin{array}{l}\text { AT } \quad 30^{\text {th }} \\
\text { Day }\end{array}$ & 62.000 & 46.000 & 220.000 & & & \\
\hline \multirow[t]{2}{*}{ Vaivarnyta } & 32 & BT $1^{\text {st }}$ Day & 3.000 & 3.000 & 4.000 & \multirow{2}{*}{-496.000} & \multirow[t]{2}{*}{0.000} & \multirow[t]{2}{*}{$<0.001 *$} \\
\hline & 32 & $\begin{array}{l}\text { AT } \quad 30^{\text {th }} \\
\text { Day }\end{array}$ & 1.000 & 1.000 & 1.000 & & & \\
\hline \multirow[t]{2}{*}{ Pidaka } & 32 & BT $1^{\text {st }}$ Day & 3.000 & 3.000 & 3.000 & \multirow{2}{*}{$-\overline{435.000}$} & \multirow[t]{2}{*}{0.000} & \multirow[t]{2}{*}{$<0.001 *$} \\
\hline & 32 & $\begin{array}{l}\text { AT } \quad 30^{\text {th }} \\
\text { Day }\end{array}$ & 1.000 & 1.000 & 2.000 & & & \\
\hline \multirow[t]{2}{*}{ Srava } & 32 & BT $1^{\text {st }}$ Day & 0.000 & 0.000 & 0.000 & \multirow[t]{2}{*}{-1.000} & \multirow[t]{2}{*}{0.000} & \multirow[t]{2}{*}{1.000} \\
\hline & 32 & $\begin{array}{l}\text { AT } \quad 30^{\text {th }} \\
\text { Day }\end{array}$ & 0.000 & 0.000 & 0.000 & & & \\
\hline \multirow[t]{2}{*}{ Shotha } & 32 & BT $1^{\text {st }}$ Day & 0.000 & 0.000 & 0.000 & \multirow[t]{2}{*}{0.000} & \multirow[t]{2}{*}{0.000} & \multirow[t]{2}{*}{1.000} \\
\hline & 32 & $\begin{array}{l}\text { AT } \quad 30^{\text {th }} \\
\text { Day }\end{array}$ & 0.000 & 0.000 & 0.000 & & & \\
\hline \multirow[t]{2}{*}{ Rukshata } & 32 & BT $1^{\text {st }}$ Day & 3.000 & 3.000 & 3.500 & \multirow{2}{*}{-496.000} & \multirow[t]{2}{*}{0.000} & \multirow[t]{2}{*}{$<0.001 *$} \\
\hline & 32 & $\begin{array}{l}\text { AT } \quad 30^{\text {th }} \\
\text { Day }\end{array}$ & 1.000 & 0.000 & 1.000 & & & \\
\hline \multirow[t]{2}{*}{ Raji } & 32 & BT $1^{\text {st }}$ Day & 3.000 & 3.000 & 3.000 & \multirow{2}{*}{$\begin{array}{l}- \\
435.000\end{array}$} & \multirow[t]{2}{*}{0.000} & $<0.001 *$ \\
\hline & 32 & $\begin{array}{l}\text { AT } \quad 30^{\text {th }} \\
\text { Day }\end{array}$ & 1.000 & 1.000 & 2.500 & & & \\
\hline
\end{tabular}


Table no.5 Unpaired T test in Group A and Group B

\begin{tabular}{|c|c|c|c|c|c|c|c|c|c|}
\hline $\begin{array}{l}\text { Subjective } \\
\text { \&Objective } \\
\text { Parameters }\end{array}$ & $\mathbf{N}$ & Day & Mean & \pm SEM & $\begin{array}{l}\text { Std } \\
\text { Dev }\end{array}$ & $\begin{array}{l}\% \\
\text { Change } \\
\downarrow\end{array}$ & Difference & $\mathbf{T}$ & $\mathbf{P}$ \\
\hline Kandu & 31 & $\begin{array}{l}\text { Group } \\
\text { A }\end{array}$ & 68.817 & \pm 4.162 & 23.171 & 69 & \multirow{2}{*}{-9.308} & \multirow{2}{*}{-1.750} & \multirow{2}{*}{0.085} \\
\hline & 32 & $\begin{array}{l}\text { Group } \\
\text { B }\end{array}$ & 78.125 & \pm 3.342 & 18.902 & $78.125 \%$ & & & \\
\hline \multirow[t]{2}{*}{ Vedana } & 31 & $\begin{array}{l}\text { Group } \\
\text { A }\end{array}$ & 3.226 & \pm 3.226 & 17.961 & 0.3 & \multirow{2}{*}{-3.024} & \multirow{2}{*}{-0.556} & \multirow{2}{*}{0.580} \\
\hline & 32 & $\begin{array}{l}\text { Group } \\
\text { B }\end{array}$ & 6.250 & \pm 4.348 & 24.593 & 6.250 & & & \\
\hline \multirow[t]{2}{*}{$\begin{array}{ll}\text { Size } & \text { of } \\
\text { Patches } & \\
\end{array}$} & 31 & $\begin{array}{l}\text { Group } \\
\text { A }\end{array}$ & 22.396 & \pm 2.158 & 12.014 & 22.4 & \multirow{2}{*}{8.074} & \multirow[b]{2}{*}{3.088} & \multirow[b]{2}{*}{0.003} \\
\hline & 32 & $\begin{array}{l}\text { Group } \\
\text { B }\end{array}$ & 64.063 & \pm 3.558 & 20.129 & 64.063 & & & \\
\hline \multirow[t]{2}{*}{ Vaivarnyta } & 31 & $\begin{array}{l}\text { Group } \\
\text { A }\end{array}$ & 55.645 & \pm 4.755 & 26.474 & 56 & \multirow{2}{*}{-8.417} & \multirow{2}{*}{-1.423} & \multirow{2}{*}{0.160} \\
\hline & 32 & $\begin{array}{l}\text { Group } \\
\text { B }\end{array}$ & 64.063 & \pm 3.558 & 20.129 & 64.063 & & & \\
\hline \multirow[t]{2}{*}{ Pidaka } & 31 & $\begin{array}{l}\text { Group } \\
\text { A }\end{array}$ & 51.651 & \pm 4.952 & 27.570 & 52 & \multirow{2}{*}{51.651} & \multirow{2}{*}{-2.754} & \multirow{2}{*}{0.697} \\
\hline & 32 & $\begin{array}{l}\text { Group } \\
\text { B }\end{array}$ & 54.405 & \pm 4.997 & 28.266 & 54.405 & & & \\
\hline \multirow[t]{2}{*}{ Srava } & 31 & $\begin{array}{l}\text { Group } \\
\text { A }\end{array}$ & 12.903 & \pm 5.225 & 29.094 & 13 & \multirow{2}{*}{68.817} & \multirow{2}{*}{78.125} & \multirow{2}{*}{0.085} \\
\hline & 32 & $\begin{array}{l}\text { Group } \\
\text { B }\end{array}$ & 2.083 & \pm 2.083 & 11.785 & 2.083 & & & \\
\hline \multirow[t]{2}{*}{ Shotha } & 31 & $\begin{array}{l}\text { Group } \\
\text { A }\end{array}$ & 0.000 & \pm 0.000 & 0.000 & 00 & \multirow{2}{*}{3.939} & \multirow[b]{2}{*}{1.209} & \multirow[b]{2}{*}{0.231} \\
\hline & 32 & $\begin{array}{l}\text { Group } \\
\text { B }\end{array}$ & 0.000 & \pm 0.000 & 0.000 & 0.000 & & & \\
\hline \multirow[t]{2}{*}{ Rukshata } & 31 & $\begin{array}{l}\text { Group } \\
\text { A }\end{array}$ & 70.430 & \pm 4.683 & 26.073 & 70 & \multirow{2}{*}{-3.528} & \multirow{2}{*}{-0.541} & \multirow{2}{*}{0.591} \\
\hline & 32 & $\begin{array}{l}\text { Group } \\
\text { B }\end{array}$ & 73.958 & \pm 4.548 & 25.729 & 73.958 & & & \\
\hline \multirow[t]{2}{*}{ Raji } & 31 & $\begin{array}{l}\text { Group } \\
\text { A }\end{array}$ & 50.707 & \pm 5.566 & 30.991 & 50.7 & \multirow{2}{*}{-6.883} & \multirow{2}{*}{-0.923} & \multirow{2}{*}{0.359} \\
\hline & 32 & $\begin{array}{l}\text { Group } \\
\text { B }\end{array}$ & 57.589 & \pm 4.976 & 28.147 & 57.589 & & & \\
\hline
\end{tabular}




\section{Specification of Shringayantra}

\section{Major Opening}

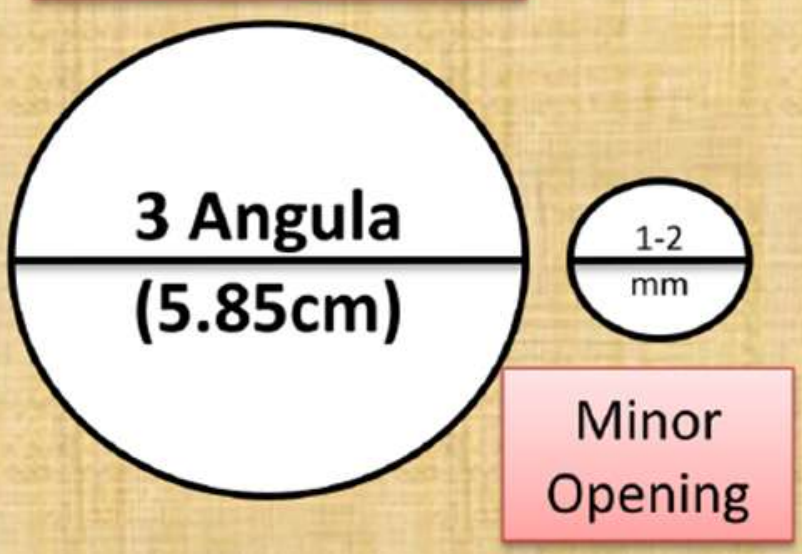

12 Angula $1-2 \mathrm{~mm}$ Long $(23.4 \mathrm{~cm})$

Specification of Shringayantra in Classics

Category

Subtype

Length

Openings

Major Opening Diameter

Minor Opening Diameter

Use

Doshaghnata

Area of working

Guna

Achushana Method

Aspiratory Force
- Yantra

- Nadiyantra

$-8 / 10 / 12$ Angula According A.H. 18 Angula

$-2$

-3 Angula

- Sarshapavata 0.5-1 cm

- Aspiration of Blood or Dushta Stanya

- Vataghna

- Twakastha Dosha (Skin and Subcutaneous tissue leval)

- Snigdha, Ushna

- Manually

- $300 \mathrm{~mm}$ of $\mathrm{Hg}$ (Average Calculated by Survey method)

Image no.1: Showing Shringayantra and its Specifications 


\section{Components of Modified Shringayantra}

Length :12 Angula Long $(23.4 \mathrm{~cm})$

Brim Circumference :3 Angula $(5.85 \mathrm{~cm})$

Minor Mouth Opening :1-2mm

\section{Furnished Shringa (Cow Horn)}

Auto Pressure Releasing Knob

Handle<smiles>[Li]</smiles>
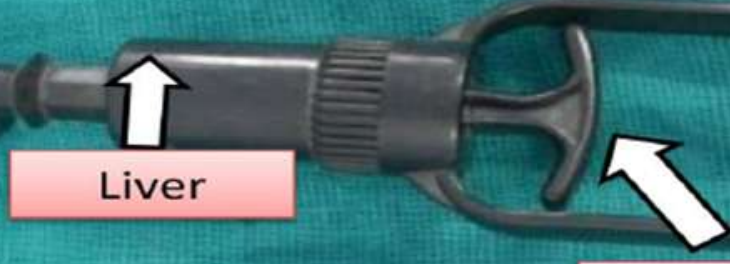

Piston

Manual Vacuum Pump

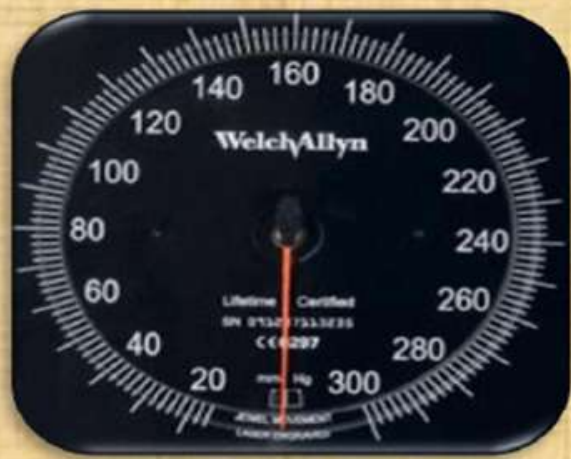

Pressure Measuring Gauge

Image 2: Component of modified Shringayantra 


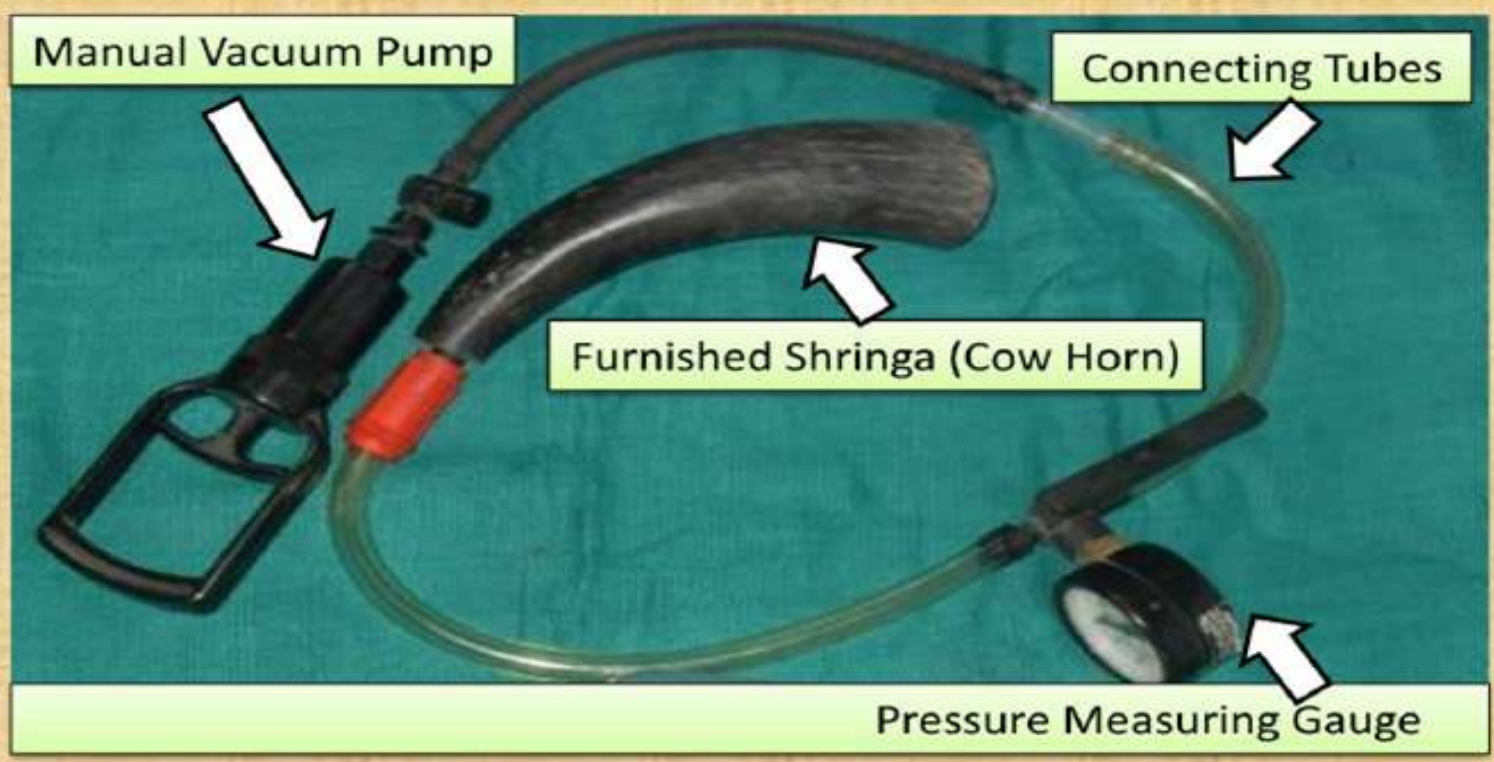

Modified Shringayantra Device

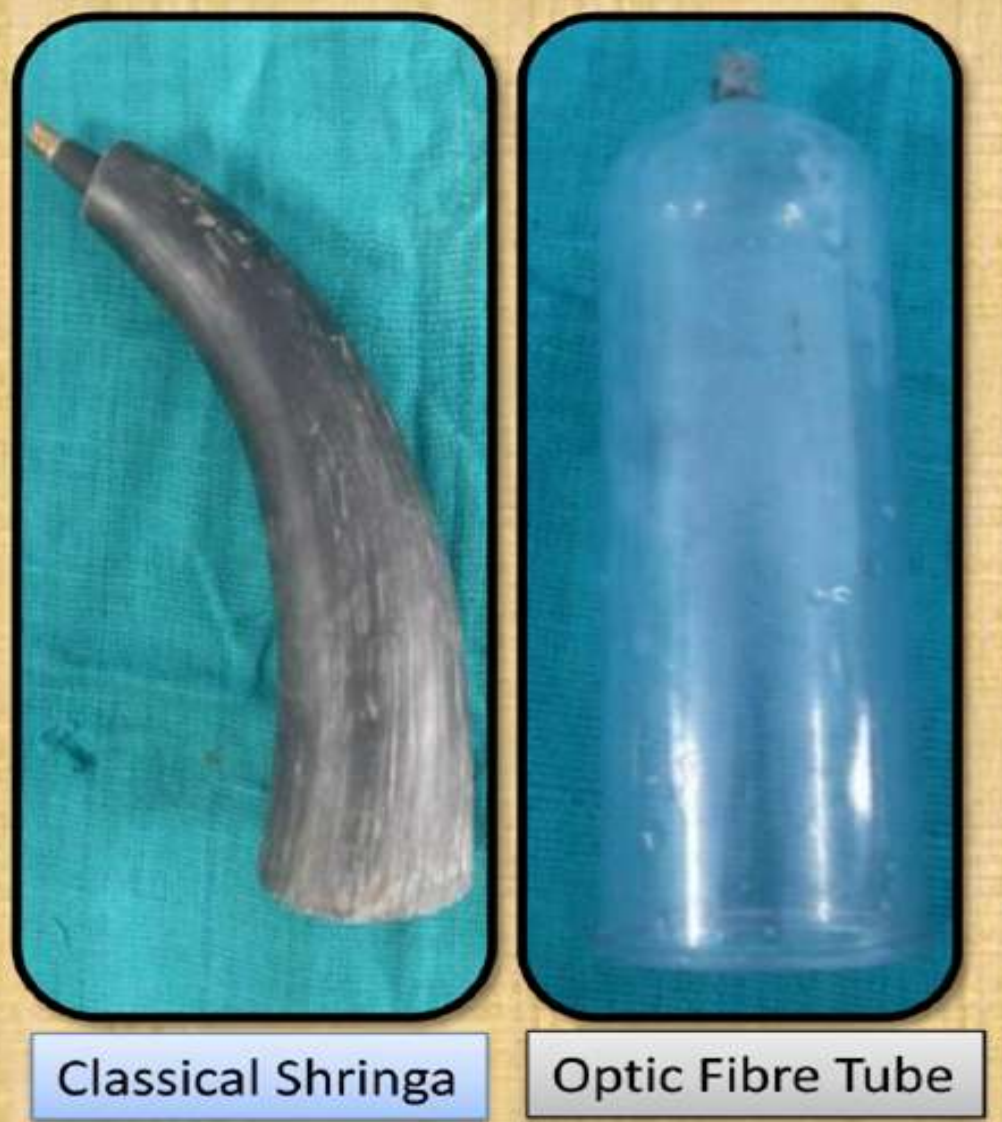

Image 3: Component of modified Shringayantra 


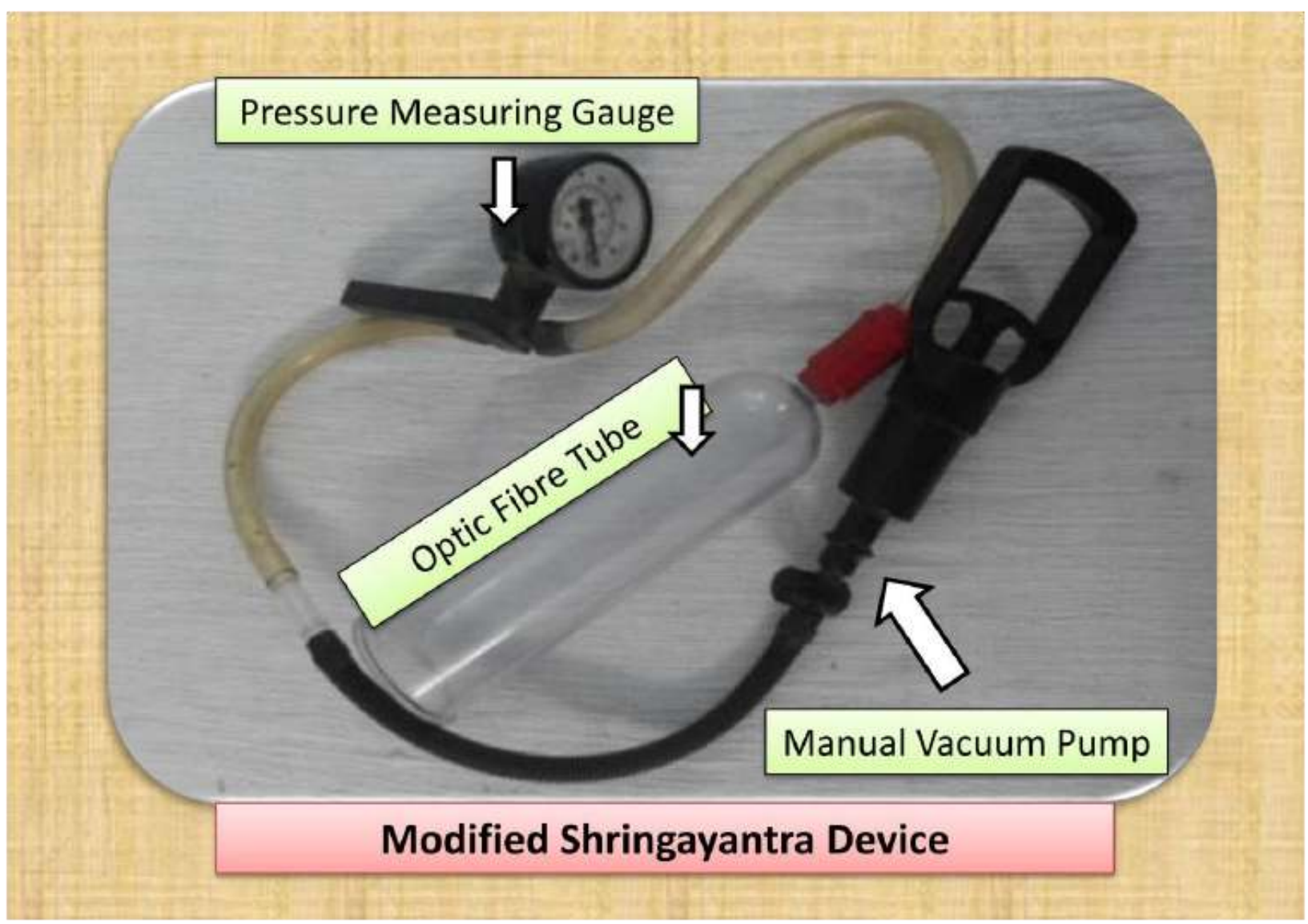

Image 4: Component of modified Shringayantra with optic Fiber Tube 